\title{
Modelling Tumor Progression, Heterogeneity, and Immune Competition
}

\author{
D. AMBROSI*, N. BELLOMO and L. PREZIOSI \\ Department of Mathematics, Corso Duca degli Abruzzi 24, Politecnico 10129, Torino, Italy
}

(Received 8 August 2000; In final form 23 April 2001)

\begin{abstract}
This paper deals with the modeling of the immune response to the evolution of the progression of endothelial cells which have lost the differentiation and start their evolution towards metastatic states. The modeling is developed in the framework of the so-called kinetic cellular theory. The model is critically analyzed on the basis of analytic solutions, asymptotic behaviors and numerical simulations that illustrate the scenarios predicted by the model. Finally, possible developments and generalizations that could describe other known phenomena are pointed out.
\end{abstract}

Keywords: Tumor; Immunitary system; Mathematical model; Progression; Kinetic cellular theory

\section{INTRODUCTION}

As a major characteristics, tumor growth and degeneration is linked to the evolution of its size and progression. A recent paper by Greller et al. (1996) has given some new ideas about the description of the maturation or progression stages of cell populations. In their phenomenologic description the stage and velocity of progression is not the same for all cells: it can vary from cell to cell, so that it is possible to introduce a suitable statistical distribution in terms of a progression variable and to study its evolution. As an example, a few cells may have a high progression, while the remaining ones may have a low progression, characterizing normal cells. The presence of progressed cells originates the competition with the immune system if it is able to recognize their degenerate characteristics. In turn, this action may be partially inhibited by cells with large progression values. Eventually, if cells reach a sufficiently high progression state they may undergo uncontrolled mitosis and condense into a solid form.

The links between the phenomenologic description proposed in Greller et al. (1996) and the mathematical model proposed in Bellomo and Forni (1994) (as well as its developments reviewed in Bellomo and De Angelis (1998)) can be immediately recognized, though the contents of the two research lines were developed independently. This paper is devoted to the deduction of a mathematical formalization consistent with the phenomenologic description given in Greller et al. (1996).

The kinetic cellular theory proposed for instance in Bellomo and Forni (1994) and Bellomo et al. (1996) provides a description of the cellular system by equations similar to those of the kinetic theory and its generalizations (Bellomo and Lo Schiavo, 1998). Specifically, this class of models defines the evolution of the distribution function over the activation states of a large population of interacting cells. The above theory can be regarded as the natural development of population dynamics models applied to the analysis of tumor growth by Gyllenberg and Webbs (1990). The specific role of various cell populations is documented in several research papers, e.g. Owen and Sherrat (1999) again on the analysis of tumor dynamics or Perelson and Weisbuch (1997) within general frameworks on the modeling of the immune response.

The paper is organized as follows:

In this introduction the aims of the paper are illustrated. "Phenomenology and scaling" section provides a resumeè of the phenomenology of the system described in Greller et al. (1996) elaborated from the viewpoint of applied mathematicians.

"A conceptual modeling framework" section deals with the design of a mathematical structure able to include a variety of models related to the above system.

*Corresponding author. 
"Some evolution models" section deals with the actual statement of the equations by proper modeling of the terms that have been introduced in the previous section.

In "Analytic solutions and asymptotic behaviors" section analytical and asymptotic solutions are shown for a large range of parameters.

The last section contains a critical analysis of the results and indicates research perspectives towards further developments.

This paper refers to the large bibliography reported in the surveys collected in Adam and Bellomo (1996) and Chaplain (1999). Additional bibliography can be found in the review paper by Bellomo and De Angelis (1998).

\section{PHENOMENOLOGY AND SCALING}

The evolution of a cell, as described by various authors, e.g. Forni et al. (1994), is regulated by the genes contained in its nucleus. These genes can be either activated or suppressed, when signals stimulate receptors on the cell surface and are then transmitted to the nucleus of the cell. The capability of receiving particular signals or the degradation of the cells can modify the usual behavior of a cell. In extreme situations, particular signals can induce a cell to reproduce itself in the form of identical descendants, the so-called clonal expansion or mitosis, or to die, the so-called apoptosis or programmed death.

Tumor cells compete with the immune system and, if not recognized and depleted, start to condense into a solid form. The solid tumor interacts with other cells through signals, which diffuse in the outer environment. The sequential steps of the evolution of the system may be summarized, from the viewpoint of a mathematician, as follows.

1. Loss of the differentiation of cells and their replication.

2. Interaction and competition at the cellular level with immune and environmental cells. This stage includes activation and inhibition of the immune system. This action is also developed through cytokine signal emission and reception which regulates cell activities.

3. Condensation of tumor cells into solid forms, macroscopic diffusion and angiogenesis.

4. Detachment of metastases and invasion.

The phenomenologic description proposed in this paper refers to the contents of Greller et al. (1996) and therefore to a large population of interacting cells characterized by a physical property called progression. Such a property is not the same for all cells, but is statistically distributed over the individuals of the population. Low progression values correspond to standard non pathologic behavior of the cell, high progression values correspond to loss of differentiation up to metastatic attitude. This means that in describing the evolution of a cell population towards pathological states a new independent variable $u$ referring to the progression states needed to be added to those usually used in discrete models (time) and in reactiondiffusion models (time and space).

Each cell may have an inner evolution from low values to large values of the progression. Still referring to the description of Greller et al. (1996), we point out that the authors leave some relevant phenomena open to investigation and interpretation (possibly within a mathematical framework). For instance, transition from a normal state of endothelial cells to a progressing state is assumed almost continuous, and the role of the contrast of the immune system is not described in details. On the other hand, the statistical distribution of the progressing state is one of the aspects which is particularly emphasized.

Qualitative behaviors of the evolution of the statistical distribution of progressing cells are given in Fig. 1, which gives two possible scenarios of progression and proliferation in metastatic states. Larger peaks refer to normal cells characterized by a smaller progression state. As time goes by, some cells may overcome a critical value, evolve and proliferate. This evolution may be contrasted by the immune system as indicated in Fig. 1a, or may not (when the action of the immune system is not sufficient) and then evolve toward metastatic states as indicated in Fig. 1b. The figure anticipates the mathematical description which is developed in what follows.

The development of mathematical models should be addressed to discuss the above phenomena and, specifically, to analyze the relevant features of the evolution with special attention to the asymptotic behavior and to the sensitivity of the parameters of the model.

\section{A CONCEPTUAL MODELING FRAMEWORK}

The aim of this section is to design a mathematical framework corresponding to the phenomenologic description given in "Phenomenology and scaling" section. We propose a system of two coupled nonlinear integrodifferential equations obtained by a balance relation used to determine the number of cells which reach a certain state either by their own maturation or by cell interactions.

Similarly to conservation equations in mechanics, this structure should be regarded as a formal one to be specialized into a specific model by means of a detailed analysis and modeling of cellular interactions. We believe that this gradual approach has some advantages. In fact, the mathematical structure can be offered to the attention of immunologists in order to improve the description of cellular interactions. Indeed, the conceptual framework should not be substantially modified by such a refinement of the modeling. The contents of this section will be then exploited, in the next one, to develop a specific model. 


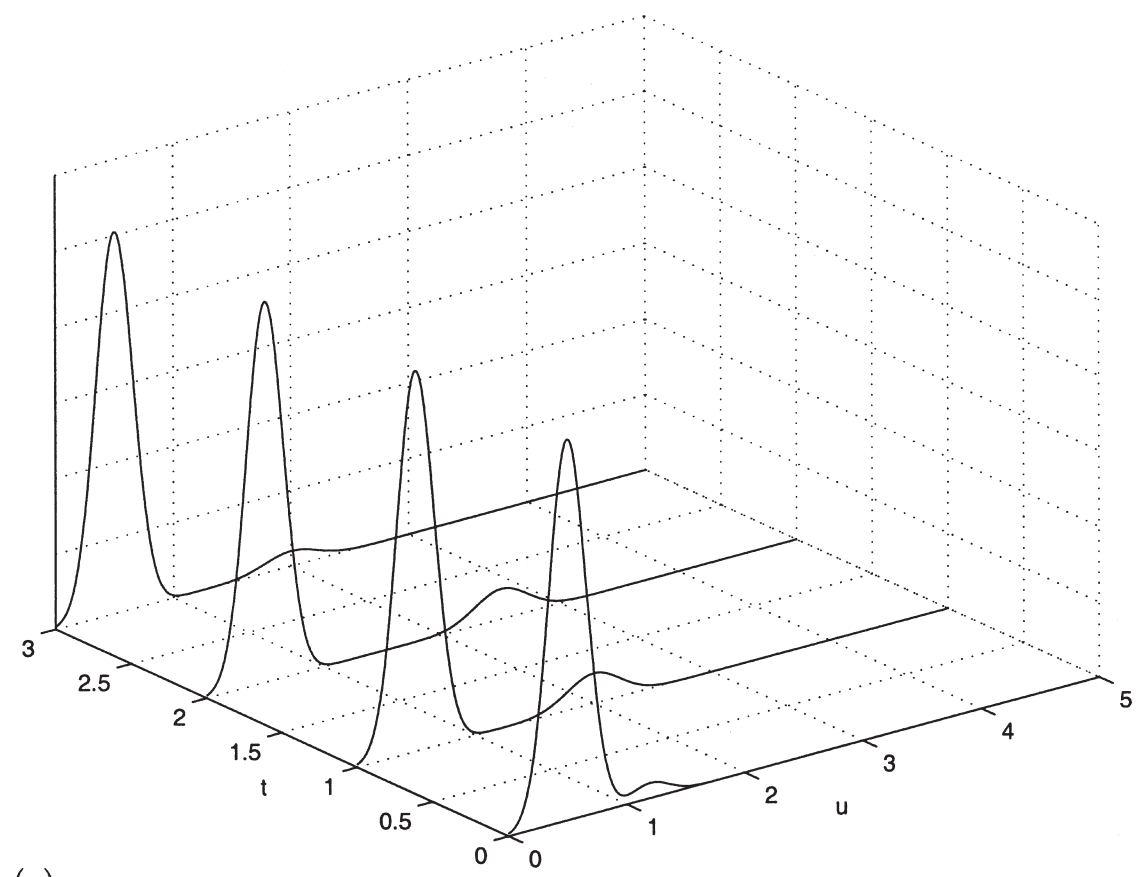

(a)

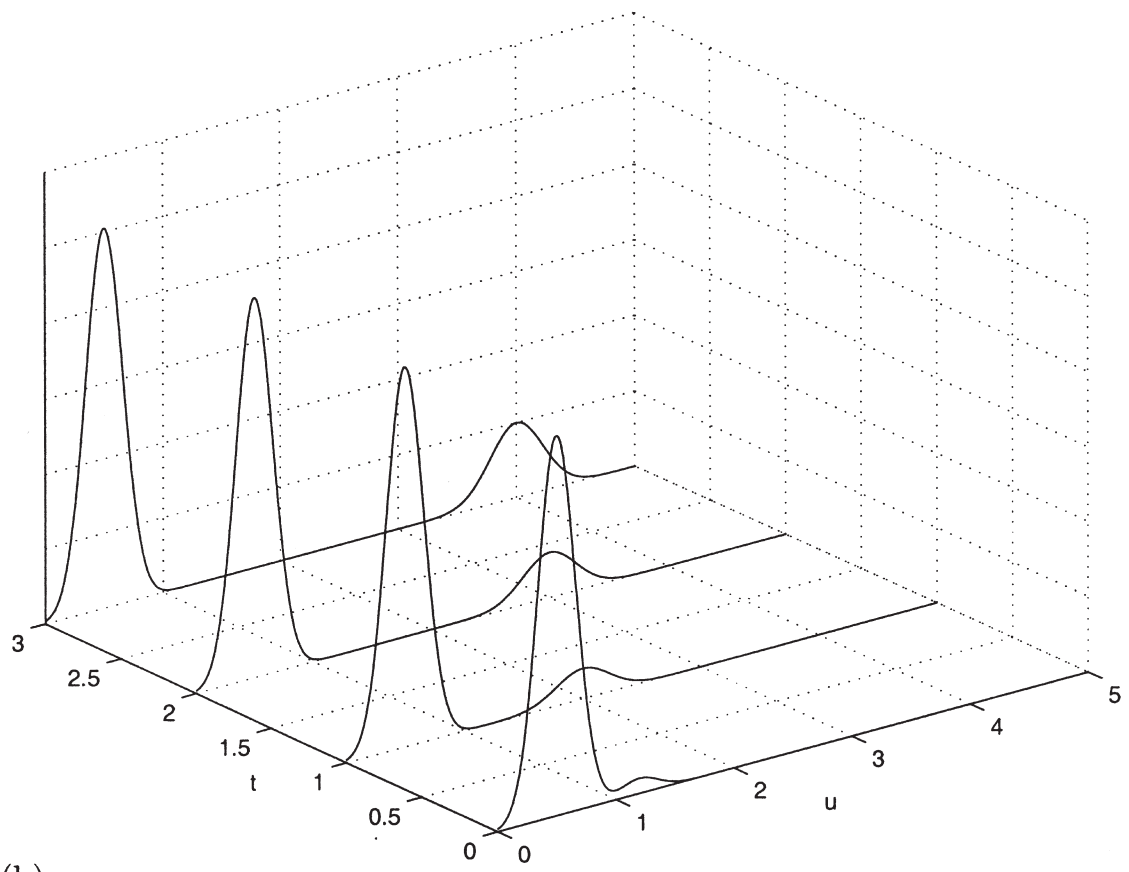

(b)

FIGURE 1 Distribution of cells over the progression state $u$ at different normalized times. Larger values of $u$ correspond to more pathological states. The smaller peaks refer to cell progressing toward more aggressive states, while the layer peaks refer to the normal cells. In (a) progression is contrasted by the immune system. In (b) it is not.

This modeling will end up with the characterization of the kernels of the integral terms of the evolution equations.

\section{Cell Populations and States}

The first step in the modeling process is the selection of the cell populations which play the game, the definition of the states which characterize them, and the type of statistical representation.

Assumption 1. (Cell Populations). The system is constituted by two interacting cell populations: environmental and immune cells, labeled, respectively, by the indexes $i=1$ and $i=2$. 
Assumption 2. (Cell State). The functional state of each cell is described by the real variable $u \in[0, \infty)$, which identifies the progression for the environmental cells and the activation for the immune cells. The progression state is conventionally divided into two regions: $D_{1}=[0,1)$ corresponding to normal cells; $D_{2}=[1, \infty)$ corresponding to progressing cells. If $u<1$ endothelial cells have no progression dynamics. On the other hand, $u \geq 1$ cells possess a certain progression velocity which may depend or not on the state $u$.

Assumption 3. (Statistical Description). The statistical description of the system is described by the distribution density functions

$$
N_{i}=N_{i}(t, u), \quad i=1,2
$$

which are such that $\mathrm{d} n_{i}=N_{i}(t, u) \mathrm{d} u$ denotes the number of cells per unit volume whose state is, at time $t$, in the interval $[u, u+\mathrm{d} u]$. Moreover,

$$
n_{i}(t)=\int_{0}^{\infty} N_{i}(t, u) \mathrm{d} u,
$$

is the number of cells of the $i$-th population at the time $t$ in a reference unit volume.

If $n_{0}$ is the number of environmental cells per unit volume at $t=0$, the following normalization of $N_{i}$ with respect to $n_{0}$ can be applied:

$$
f_{i}(t, u)=\frac{1}{n_{0}} N_{i}(t, u) .
$$

The number of cells per unit volume which at time $t$ have a progression state in $\left[u_{1}, u_{2}\right]$ is then

$$
n_{i}^{\left[u_{1}, u_{2}\right]}=n_{0} \int_{u_{1}}^{u_{2}} f_{i}(t, u) \mathrm{d} u .
$$

\section{Cellular Interactions}

We consider the description of a general framework for the modeling of cell interactions in view of the design of a mathematical model suitable to define the evolution of the distribution functions $f_{i}$.

Assumption 4. The intrinsic progression and activation of the $i$-th population is defined by means of the progression velocity $c_{i}(u)$ which describes the evolution of the cell population in absence of other interactions. It is such that under this condition in the interval $[t, t+\mathrm{d} t]$ the cell of the $i$-th population changes its state from $u$ to $u+\mathrm{d} u=u+c_{i}(u) \mathrm{d} t$.
Assumption 5. In addition to the natural progression, the state of a cell or the number of cells with state $u$ can change because of

i) Mass conservative interactions between pairs of cells, i.e. interactions which are not responsible for proliferation or destruction of cells but only of a change in the activation state for one or both interacting cells.

ii) Proliferative-destructive interactions between cell pairs;

iii) External sources or sinks of cells (or input/output), e.g. production of immune cells by the bone marrow, possibly pharmacologically stimulated, destruction of tumor cells by medical treatment, injection of cells.

Assumption 6. The evolution due to conservative encounters modifies the progression of tumor cells and the activation of immune cells. Cell interactions in the case of mass conservative encounters will be defined by means of two physical quantities: the encounter rate $\eta_{i j}$ and the transition probability density $\psi_{i j}$. In more detail, conservative encounters between the cell of the $i$-th population with state $v$ and the cell of the $j$-th population with state $w$ are quantitatively described by the transition rate

$$
T_{i j}(v, w ; u)=\eta_{i j}(v, w) \psi_{i j}(v, w ; u),
$$

where $n_{i j}(v, w)$ denotes the number of encounters per unit volume and unit time between cell pairs of the $(i, j)$-th populations with states $v$ and $w$, respectively, and $\psi_{i j}(v, w ; u)$ denotes the probability of transition of the $i$ th cell to the state $u$, given its initial state $v$ and the state $w$ of the encountering cells belonging to the $j$-th population. Hence, $T_{i j}(v, w ; u)$ denotes the number of encounters per unit volume and unit time between cell pairs of the $(i, j)$-th populations with states $v$ and $w$, respectively, with transition of the $i$-th cells into the state $u$.

Assumption 7. Proliferative encounters will be described by two quantities: the proliferation rate $p_{i j}$ and the proliferation probability density $\varphi_{i j}$. These encounters occur between cell pairs belonging to the same or to different populations, and generate new cells in one or both populations. These interactions are quantitatively described by the proliferation transition rate

$$
P_{i j}(v, w ; u)=p_{i j}(v, w) \varphi_{i j}(v, w ; u),
$$

where $p_{i j}(v, w)$ denotes the number of cells produced per unit volume and unit time due to the encounters of cell pairs of the $(i, j)$-th populations with states $v$ and $w$, respectively, and $\varphi_{i j}(v, w, u)$ is the probability density of proliferation of the $i$-th cell in the state $u$ by encounters of cells belonging to the $i$-th and $j$-th populations with state $v$ and $w$, respectively, in the following. Hence, $P_{i j}(v, w, u)$ 
denotes the number of $i$-th cells in the state $u$ per unit volume and unit time which proliferate because of the encounters between cell pairs of the $(i, j)$-th populations with states $v$ and $w$, respectively. It is assumed that

$$
\varphi_{i j}(v, w ; u)=\delta(v-u),
$$

that is daughter cells inherit the same activation state as the mother cells.

Assumption 8. Destructive encounters occur between cell pairs of different populations, and generate a destruction in one or both of them. These interactions are quantitatively described by the destruction rate $d_{i j}(u, w)$, which is the number of $i$-th cells with state $v$ destroyed as the result of the interaction with $j$-th cells with state $w$.

The evolution equation obtained using the above assumptions consists in the following system of two coupled integro-differential equations:

$$
\begin{aligned}
& \frac{\partial f_{i}}{\partial t}(t, u)+\frac{\partial}{\partial u}\left[c_{i}(u) f_{i}(t, u)\right] \\
& \quad=\sum_{i=1}^{2}\left(\mathscr{G}_{i j}^{*}-\mathscr{L}_{i j}^{*}+\mathscr{G}_{i j}-\mathscr{L}_{i j}\right)(t, u)+\mathscr{S}_{i}(t, u),
\end{aligned}
$$

where

$$
\begin{gathered}
\mathscr{G}_{i j}^{*}=\int_{0}^{\infty} \int_{0}^{\infty} \eta_{i j}(v, w) \psi_{i j}(v, w ; u) f_{i}(t, v) f_{j}(t, w) \mathrm{d} v \mathrm{~d} w \\
\mathscr{L}_{i j}^{*}=f_{i}(t, u) \int_{0}^{\infty} \eta_{i j}(u, w) f_{j}(t, w) \mathrm{d} w \\
\mathscr{G}_{i j}=f_{i}(t, u) \int_{0}^{\infty} p_{i j}(u, w) f_{j}(t, w) \mathrm{d} w
\end{gathered}
$$

and

$$
\mathscr{L}_{i j}=f_{i}(t, u) \int_{0}^{\infty} d_{i j}(u, w) f_{j}(t, w) \mathrm{d} w,
$$

for $i, j=1,2$.

The terms $\mathscr{G} *$ and $\mathscr{L} *$ correspond to the gain and loss of cells in the state $u$ due to conservative encounters, respectively. The terms $\mathscr{G}$ and $\mathscr{L}$ correspond to the gain and loss of cells due to proliferative and destructive encounters, respectively. Finally, the source term $\mathscr{S}_{i}$ models external input, e.g. production from bone marrow and natural death of cells.

The above model defines a framework for continuous distribution functions. A slightly simpler framework can be developed assuming that the state of the immune cells is not continuously distributed, but simply concentrated on two states: the active and inhibited ones. In this case, the evolution of the immune cells is simply identified by the number densities $n_{2}=n_{2}(t)$, corresponding to active cells, and $n_{3}=n_{3}(t)$, corresponding to inhibited immune cells.

Considering that inhibited cells do not contribute to the evolution of the progression factor, the model can refer to $f_{1}$ and $n_{2}$ only. This new model will be called semicontinuous. The various terms defined for the continuous framework assume a slightly different meaning which will be defined later.

The new mathematical framework consists of the following system of coupled equations:

$$
\begin{aligned}
& \frac{\partial f_{1}}{\partial t}(t, u)+\frac{\partial}{\partial u}\left[c_{1}(u) f_{1}(t, u)\right] \\
& =\int_{0}^{\infty} \int_{0}^{\infty} \eta_{11}(v, w) \psi_{11}(v, w ; u) f_{1}(t, v) f_{1}(t, w) \mathrm{d} v \mathrm{~d} w \\
& \quad-f_{1}(t, u) \int_{0}^{\infty} \eta_{11}(u, w) f_{1}(t, w) \mathrm{d} w \\
& \quad+\int_{0}^{\infty} \eta_{12}(v) \psi_{12}^{*}(v ; u) f_{1}(t, v) \mathrm{d} v n_{2}(t) \\
& \quad-\eta_{12}(u) f_{1}(t, u) n_{2}(t) \\
& \quad+f_{1}(t, u) \int_{0}^{\infty} \mu_{11}(u, w) f_{1}(t, w) \mathrm{d} w \\
& \quad+f_{1}(t, u) \mu_{12}(u) n_{2}(t)+\mathscr{S}_{1}(t, u)
\end{aligned}
$$

and

$$
\begin{aligned}
\frac{\mathrm{d} n_{2}}{\mathrm{~d} t}(t)= & \int_{0}^{\infty} \eta_{21}(w) \psi_{21}^{*}(w) f_{1}(t, w) \mathrm{d} w n_{2}(t) \\
& -n_{2}(t) \int_{0}^{\infty} \eta_{21}(w) f_{1}(t, w) \mathrm{d} w \\
& +\eta_{22} \psi_{22}^{*} n_{2}(t) n_{2}(t)-\eta_{22} n_{2}(t) n_{2}(t) \\
& +n_{2}(t) \int_{0}^{\infty} \mu_{21}(w) f_{1}(t, w) \mathrm{d} w \\
& +\mu_{22} n_{2}(t) n_{2}(t)+\mathscr{S}_{2}(t, u)
\end{aligned}
$$

where

$$
\mu_{i j}(\cdot)=p_{i j}(\cdot)-d_{i j}(\cdot),
$$

is the net proliferation rate, or

$$
\begin{aligned}
& \frac{\partial f_{1}}{\partial t}(t, u)+\frac{\partial}{\partial u}\left[c_{1}(u) f_{1}(t, u)\right] \\
& \quad=\int_{0}^{\infty} \int_{0}^{\infty} \eta_{11}(v, w) \psi_{11}(v, w ; u) f_{1}(t, v) f_{1}(t, w) \mathrm{d} v \mathrm{~d} w \\
& \quad+f_{1}(t, u) \int_{0}^{\infty}\left[\mu_{11}(u, w)\right. \\
& \left.\quad-\eta_{11}(u, w)\right] f_{1}(t, w) \mathrm{d} w \\
& \quad+n_{2}(t) \int_{0}^{\infty} \eta_{12}(v) \psi_{12}^{*}(v ; u) f_{1}(t, v) \mathrm{d} v \\
& \quad+\left[\mu_{12}(u)-\eta_{12}(u)\right] f_{1}(t, u),
\end{aligned}
$$


and

$$
\begin{aligned}
\frac{\mathrm{d} n_{2}}{\mathrm{~d} t}(t)= & \int_{0}^{\infty}\left[\mu_{21}(w)-\eta_{21}(w) \times(1\right. \\
& \left.\left.-\psi_{21}^{*}(w)\right)\right] f_{1}(t, w) \mathrm{d} w n_{2}(t)+\left[\mu_{22}\right. \\
& \left.-\eta_{22}\left(1-\psi_{22}^{*}\right)\right] n_{2}^{2}(t) .
\end{aligned}
$$

The terms $\psi_{12}^{*}, \psi_{21}^{*}$ and $\psi_{22}^{*}$ have a slightly different meaning than $\psi_{12}, \psi_{21}$ and $\psi_{22}$, directly related to the semicontinous character of the model. For instance, $\psi_{21}^{*} \leq$ 1 represents the probability that an immune cell, after interaction with a progressed cell, remains active, while $1-\psi_{21}^{*}$ represents the probability that an immune cell, after interaction, moves into the population of the inhibited immune cells.

Both classes of models represent a rather general framework which can include the descriptions summarized in "Phenomenology and scaling". The specific models proposed in "Some evolution model" will be developed by relatively less general assumptions which require, as we shall see, a detailed analysis and simulation of cell interactions.

Moreover, we recall that the idea of discretizing the cell states in the kinetic cellular theory was first introduced by Lo Schiavo (1996). This idea is now developed by various authors Arlotti et al. (1999). We also point out that the model represented by Eqs. (3.12a) and (3.12b) includes transition from one population to the other. The mathematical framework for such a class of models was first proposed in Arlotti et al. (2000).

\section{SOME EVOLUTION MODELS}

The general framework described in "A conceptual modeling framework" section can generate specific models after a detailed modeling of cell interactions. Operating in the framework of semicontinuous models here we develop some simple models related to all type of interactions described in the preceding section and represented in Fig. 2. More specifically, while evolving towards metastatic states, progressing cells interact with other cells of the body. The interaction with the immune system may generate both the death of the progressing cells and a decrease in the progression state. The interaction with other environmental cells favors proliferation, e.g. because capillaries bring the necessary nutrient. On the other hand, cells of the immune system react to the presence of progressing cells by proliferating. However, the interaction with tumor cells may inactivate or kill an immune cell. In the following the previous scenario is put in mathematical terms. However, an effort is made to keep reasonably lower the number of parameters. Specifically the framework is simplified assuming that $\mathscr{S}_{i}=0$, i.e. the inlet from bone marrow equates the natural death of cells

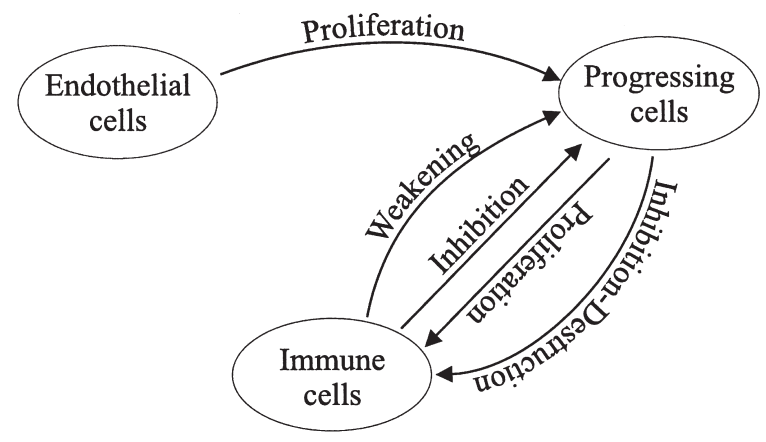

FIGURE 2 Cellular activities and interactions. Normal endothelial cells contribute to the proliferation of progressing cells. Progressing cells try to inhibit the immune system while immune cells proliferate when they recognize progressing cells and fight against them.

and the output of interactions linearly depend on the state of the interacting pairs.

The aim of this first proposal consists of obtaining an immediate description of tumor progression and immune competition to be analyzed at a quantitative level and compared with experimental data. The model can certainly be improved and refined following the critical analysis developed in the previous section.

It is useful to introduce the stepwise function:

$$
\begin{gathered}
U_{[a, b]}(z): U_{[a, b]}(z)=1 \quad \text { if } z \in[a, b] ; \\
U_{[a, b]}(z)=0 \quad \text { if } z \notin[a, b],
\end{gathered}
$$

which will be used in the calculations which follow.

\section{Progression Velocity}

Environmental cells which, for genetic modifications, reach a state larger that the critical value $u=1$ start progressing toward large values of $u$ with velocity $c_{1}$. On the other hand, if $u \leq 1$, the progression velocity is equal to zero: $c_{1}=0$.

The simplest modeling of the above feature is

$$
c_{1}:=c U_{[1, \infty)}(u) .
$$

In general, it is reasonable to assume that only a small number of cells are initially in the progression region, namely

$$
\varepsilon=\int_{1}^{\infty} f_{1}(0, u) \mathrm{d} u \ll 1 .
$$

\section{Encounter Rate}

It is assumed that all encounter rates are constant for all interacting pairs and that

$$
\eta_{i j}=\eta=1, \quad \forall i, j=1,2 .
$$


Hence we shall put the above constant equal to one, that is equivalent to include the encounter rate into the time scale.

\section{Conservative Encounters}

Consider separately all types of conservative encounters. The denomination non trivial will be used to indicate those encounters which modify the activation state of the interacting cells.

Referring to conservative encounters of environmental cells with other (either environmental or immune) cells, the specialization of the probability densities $\psi_{11}$ and $\psi_{12}^{*}$ are assumed to be delta functions

$$
\begin{aligned}
\psi_{11}(v, w ; u) & =\delta\left(u-m_{11}(v, w)\right), \quad \text { and } \\
\psi_{12}^{*}(v ; u) & =\delta\left(u-m_{12}(v)\right),
\end{aligned}
$$

where $m_{1 j}$ corresponds to the output which may depend on the activations of the interacting pairs. In more detail, we assume the following.

Assumption 1. Environmental cells (progressed or not) do not change their state when encountering other cells of the same population. If the environmental cell is not progressed, its state does not change when it encounters a cell of the immune system. If a progressed cell encounters an active immune cell, its state decreases, but cannot fall beneath $u=1$.

This assumption can be formalized as follows:

$$
\psi_{11}(v, w ; u)=\delta(u-v)
$$

and

$$
\begin{aligned}
\psi_{12}(v ; u)= & \delta(u-v) U_{[0,1)}(v)+U_{[1, \infty)}(v) \times \delta(u \\
& \left.-\left(v-\alpha_{12}(v-1)\right)\right),
\end{aligned}
$$

where $0 \leq \alpha_{12}<1$.

Referring to conservative encounters of immune cells the only encounters with non trivial output are those between active immune cells and progressed cell. The result consists in producing an inhibited immune cell with a probability $1-\beta_{21}$, where $\beta_{21} \in[0,1]$ is the probability to remain active after the encounter:

$$
\begin{aligned}
\psi_{21}^{*}(w) & =1 \cdot U_{[0,1)}(w)+\left(1-\beta_{21}\right) U_{[1, \infty)}(w), \\
\psi_{22}^{*} & =1 .
\end{aligned}
$$

\section{Proliferative and Destructive Encounters}

As above, we consider separately all types of proliferative and destructive encounters. The denomination non trivial will now be used to indicate those encounters which generate a non vanishing net proliferation rate not equal to zero.

- The net proliferation rate of endothelial cells with activation state less than the critical value $u=1$, due to encounters with other endothelial cells, is equal to zero. On the other hand, when an endothelial cell falls into the progressing state larger than one, it undergoes uncontrolled mitosis generated by encounters with nonprogressed cells, proliferation is assumed to be proportional to $u-1$ so that one has a net proliferation rate

$$
\mu_{11}(u, w)=\gamma_{11}(u-1) U_{[1, \infty)}(u) U_{[0,1]}(w) .
$$

- The interaction of a progressed cell, with state larger than one, with the active immune system produces a death rate independent of the status of the cell. The net proliferation rate is equal to zero if the state is less than one

$$
\mu_{12}(u)=-\delta_{12} U_{[1, \infty)}(u) .
$$

- The interaction of active immune cells with progressed cells produces a net proliferation rate greater than zero if the state of the progressed cells is greater than one.

$$
\mu_{21}(w)=\gamma_{21} U_{[1, \infty)}(w) .
$$

\section{Evolution Models}

The qualitative behavior of the solution of the general model outlined in the previous section is not modified in essence by some simplifying assumptions. For instance a delta function can be used to represent conservative interactions, so that double integrals reduce to simple integrals and analytic solutions can be obtained for some special cases. Actually, one may expect additional stochasticity, e.g. variance larger than zero, but the behavior of the solution is not modified significantly by such an assumption.

Based on the above modeling of cell interactions, we are able to derive the evolution equation. Technical calculations lead to the following result for $u<1$ :

$$
\frac{\partial f_{1}}{\partial t}=0 .
$$


On the other hand, for $u \geq 1$ the model writes

$$
\begin{aligned}
& \frac{\partial f_{1}}{\partial t}(t, u)+c \frac{\partial f_{1}}{\partial u}(t, u)=n_{2}(t) \\
& \quad \times \frac{1}{1-\alpha_{12}} f_{1} t, \frac{u-\alpha_{12}}{1-\alpha_{12}}-f_{1}(t, u) \\
& \quad+\gamma_{11}(1-\varepsilon)(u-1) f_{1}(t, u) \\
& \quad-\delta_{12} n_{2}(t) f_{1}(t, u), \\
& \frac{\mathrm{d} n_{2}}{\mathrm{~d} t}(t)=-\beta_{21} n_{2}(t) \int_{1}^{\infty} f_{1}(l, u) \mathrm{d} u \\
& \quad+\gamma_{21} n_{2}(t) \int_{1}^{\infty} f_{1}(t, u) \mathrm{d} u .
\end{aligned}
$$

and is characterized by six phenomenologic parameters:

$c$ is the progression velocity of endothelial cells. It appears only in the equations describing the critical region $u>1$. The case $c=0$ corresponds to non-progressing cells, i.e. not evolving toward metastatic states.

$\alpha_{12}$ is the parameter corresponding to the ability of the active immune cells to reduce the progression of endothelial cells in the critical region $u>1$. The parameter takes values in $[0,1)$. The value $\alpha_{12} \approx 1$ corresponds to maximum weakening ability, while $\alpha_{12}=$ 0 to total lack of such an ability.

$\beta_{21}$ is the parameter corresponding to the ability of progressing cells to inhibit the immune cells. The parameter takes values in $[0,1]$. For $\beta_{21}=1$ one has maximum inhibition ability, while $\beta_{21}=0$ corresponds to lack of inhibition ability.

$\gamma_{11}$ is the proliferation rate of progressing cells due to their interaction with endothelial cells with state $u$ smaller than one.

$\gamma_{21}$ is the proliferation rate of immune cells due to their interaction with progressing cells.

$\delta_{12}$ is the (positive) parameter corresponding to the ability of active immune cells to destroy progressing cells.

Before proceeding further in the discussion of the qualitative behavior of the solution of Eq. (4.12), it is useful to observe from Eq. (4.11), where one can focus on the evolution of progressed cells only $(u \geq 1)$ introducing the variable $z=u-1$ and rewriting the set of equations as follows

$$
\begin{aligned}
& \frac{\partial f_{1}}{\partial t}(t, z)+c \frac{\partial f_{1}}{\partial z}(t, z)= n_{2}(t)\left[\alpha f_{1}(t, \alpha z)-(1\right. \\
&\left.+\delta) f_{1}(t, z)\right]+\gamma z f_{1}(t, z), \\
& \frac{\mathrm{d} n_{2}}{\mathrm{~d} t}(t)=\nu n_{2}(t) \int_{0}^{\infty} f_{1}(t, z) \mathrm{d} z,
\end{aligned}
$$

where

$$
\begin{gathered}
\alpha=\frac{1}{1-\alpha_{12}}, \quad \gamma=\gamma_{11}(1-\varepsilon), \\
\nu=\gamma_{21}-\beta_{21}, \quad \delta=\delta_{12} .
\end{gathered}
$$

The analysis carried out in the sequel of the paper and all related figures will then focus on the evolution of the progressing tail of the distribution function, that is the smaller peaks drawn in Fig. 1.

Referring to the parameters listed in Eq. (4.14) the following remarks can be made.

i) The parameter $\alpha$ is such that $\alpha \geq 1$, larger values of $\alpha$ corresponding to greater ability of immune cells to control the progression of endothelial cells.

ii) The parameter $\nu \geq-1$ vanishes when the interaction of immune cells with progressed cells generates a balance between the proliferation rate and the inhibition-destruction rate caused by progressed cell. In this case $n_{2}$ remains constant in time. When these two phenomena are not balanced $\nu \neq 0$. In particular, if inhibition is stronger than proliferation $\nu<0$ and $n_{2}$ decreases in time.

The first equation in (4.13) is characterized by a hyperbolic operator on the left hand side and a nonlocal term on the right hand side. The latter term affects the evolution in time of $f_{1}(t, z)$ through the evolution at higher value of progression $(\alpha z \geq z)$. This implies that

$$
\begin{array}{r}
f_{1}(t=0, z)=0, z>z_{s} \Rightarrow \\
f_{1}(t, z)=0, \\
\forall z>z_{s}+c t,
\end{array}
$$

i.e. if the initial distribution has bounded support in $\left[0, z_{s}\right]$, the solution has bounded support for any finite time, too. Integrating Eq. (4.13) over $z$ yields

$$
\frac{\mathrm{d} F}{\mathrm{~d} t}(t)=c b(t)+\gamma F_{1}(t)-\delta n_{2}(t) F(t)
$$

where

$$
F(t)=\int_{0}^{\infty} f_{1}(t, z) \mathrm{d} z
$$

is related to the total number of progressive cells,

$$
F_{1}(t)=\int_{0}^{\infty} z f_{1}(t, z) \mathrm{d} z
$$

is the first moment of $f_{1}$, and $b(t)$ is the inflow at $z=0$, i.e. the degeneration of endothelial cells in progressing cells.

In addition to $F(t) \equiv F_{0}(t)$, further information on the progression can be obtained by looking at the evolution of 
higher moments:

$$
F_{k}(t)=\int_{0}^{\infty} z^{k} f_{1}(t, z) \mathrm{d} z
$$

A hierarchy of evolution equations for the moments can be obtained integrating Eq. (4.13) times $z^{k}$.One then has

$$
\begin{gathered}
\frac{\mathrm{d} F_{k}}{\mathrm{~d} t}(t)=c k F_{k-1}(t)+\left(\frac{1}{\alpha^{k}}-1-\delta\right) n_{2}(t) F_{k}(t) \\
+\gamma F_{k 1}(t), \quad k=1,2,3, \ldots
\end{gathered}
$$

In more detail, with an abuse of terminology, one can define the mean progression and standard deviation (or second central moment) as

$$
\begin{gathered}
m(t)=\frac{F_{1}(t)}{F(t)} \\
\sigma(t)=\left[\frac{F_{2}(t)}{F(t)}-m^{2}(t)\right]^{1 / 2} .
\end{gathered}
$$

Taking for sake of simplicity $b(t)=0$, one can then rewrite Eq. (4.16) and the first two equations of the hierarchy (4.20) in terms of the following system of ordinary differential equations:

$$
\begin{aligned}
\frac{\mathrm{d} F}{\mathrm{~d} t} & =\left(\gamma m-\delta n_{2}\right) F, \\
\frac{\mathrm{d} m}{\mathrm{~d} t} & =c-1-\frac{1}{\alpha} n_{2} m+\gamma \sigma^{2}, \\
\frac{\mathrm{d} \sigma^{2}}{\mathrm{~d} t} & =-1-\frac{1}{\alpha^{2}} \sigma^{2}+1-\frac{1^{2}}{\alpha} m^{2} n_{2}+\gamma F_{3}^{c},
\end{aligned}
$$

where

$$
F_{3}^{c}(t)=\frac{1}{F(t)} \int_{0}^{\infty}[z-m(t)]^{3} f_{1}(t, z) \mathrm{d} z
$$

We remark that the above system (4.23) is not a closed set of equations, unless $\gamma=0$.

\section{ANALYTIC SOLUTIONS AND ASYMPTOTIC BEHAVIORS}

In order to provide a qualitative description of the role of the parameters in the evolution, we first consider some simpler cases and then discuss some aspects of the asymptotic behavior in the general case. Indeed, considering that the full problem is nonlinear (its solution is not a simple superposition of separate effects), the analysis of simpler models is useful without any doubt. In fact, a description of the behavior of the solution in simple configurations, when some biological effects are negligible, suggests which are the crucial parameters to be investigated by asymptotic analysis or numerical simulation in the general case. Last, but not least, exact solutions provide test cases for computer simulations.

Specifically, focusing on progressing cells $(z>0)$ only, we consider the following particular cases.

Case I. $\alpha=1, \gamma=\nu=\delta=0$

This trivial case corresponds to no influence of the immune system on the progressing cells and a balance between proliferation and inhibition of immune cells. This implies that $n_{2}$ is constant and $f_{1}$ evolves according to

$$
\frac{\partial f_{1}}{\partial t}+c \frac{\partial f_{1}}{\partial z}=0
$$

which means that the initial distribution "progresses" unchanged along the characteristics $z-c t=$ const. Referring to Eq. (4.23), (if $b(t)=0$ ) then $F=$ const, $m(t)=m_{0}+c t$ and $\sigma=$ const, that is the mean progression state of the cell population constantly increases, though the total number of cells remains constant.

Case II. $\alpha>1, \gamma=\nu=\delta=0$

In this case the unique action of the immune system on the progressing cells is the conservative control of its progression. In addition, a balance between proliferation and inhibition of immune cells occurs and tumor cells neither proliferate nor are destroyed. Again, $n_{2}$ has a constant value but $f_{1}$ evolves according to the non local equation

$$
\frac{\partial f_{1}}{\partial t}+c \frac{\partial f_{1}}{\partial z}=n_{2}\left[\alpha f_{1}(t, \alpha z)-f_{1}(t, z)\right] .
$$

From Eq. (4.23) one has that (if $b(t)=0) F(t)$ is constant and

$$
\begin{gathered}
m(t)=\frac{\alpha c}{(\alpha-1) n_{2}}+C \exp -1-\frac{1}{\alpha} n_{2} t, \\
\sigma^{2}(t)=A+\left(\sigma_{0}^{2}-A-2 \alpha \frac{c}{n_{2}} C+C^{2}\right) \exp -1 \\
-\frac{1}{\alpha^{2}} n_{2} t+2 \alpha \frac{c}{n_{2}}+C \exp -21-\frac{1}{\alpha} n_{2} t \\
-C^{2} \exp -2-\frac{1}{\alpha} n_{2} t,
\end{gathered}
$$

where

$$
A=\frac{\alpha^{2} c^{2}}{\left(\alpha^{2}-1\right) n_{2}^{2}}, \quad C=m_{0}-\frac{\alpha c}{(\alpha-1) n_{2}},
$$

and $m_{0}$ and $\sigma_{0}$ are the initial values of $m(t)$ and $\sigma(t)$. 
It can be noticed that as $t \rightarrow \infty, m(t)$ and $\sigma(t)$ tend respectively to the constants

$$
m^{\infty}=\frac{\alpha}{(\alpha-1)} \frac{c}{n_{2}}, \quad \sigma^{\infty}=\frac{\alpha}{\sqrt{\alpha^{2}-1}} \frac{c}{n_{2}}
$$

independently of the initial data.

Actually, from Eq. (4.20) one can observe that all moments $F_{k}$ tend to

$$
F_{k}^{\infty}=\frac{k ! \alpha^{k(k+1) / 2}}{\prod_{j=1}^{k}\left(\alpha^{j}-1\right)} \frac{c^{k}}{n_{2}^{k}} \bar{F},
$$

where $\bar{F}$ is the constant value of $F$. This suggests the possibility that Eq. (5.2) admit steady state solutions. In fact, the source term on the right hand side of Eq. (5.2) acts a backscattering of the unknown $f_{1}$ at a backward distance $z / \alpha$. The steady state solution is then characterized by a balance between transport and backscattering effect. The steady state solutions $f_{1}^{\infty}$ satisfy the ordinary differential equation

$$
\frac{1}{\lambda} \frac{\mathrm{d} f_{1}^{\infty}}{\mathrm{d} z}+f_{1}^{\infty}=\alpha f_{1}^{\infty}(\alpha z)
$$

where $\lambda=n_{2} / c$

The solution $f_{1}^{\infty}$ can be represented in terms of a set of functions that characterizes the solution of the standard exponential-rate equations. By the set of functions $(-1)^{k} \mathrm{e}^{-\lambda \alpha^{k} z}$ we can represent the function $f_{1}^{\infty}$ as

$$
f_{1}^{\infty}(z)=\sum_{k=0}^{\infty}(-1)^{k} A_{k} \mathrm{e}^{-\lambda \alpha^{k} z}
$$

Substituting the latter expression into the differential Eq. (5.3) one obtains that the amplitudes $A_{k}$ are related by the recursive relation

$$
A_{k}=\frac{\alpha}{\alpha^{k}-1} A_{k-1}, \quad \forall k \geq 1,
$$

so that any amplitude can be written in terms of the zero-th one as follows:

$$
A_{k}=\frac{\alpha^{k}}{\prod_{j=1}^{k}\left(\alpha^{j}-1\right)} A_{0}, \quad \forall k \geq 1 .
$$

The steady state solution $f_{1}^{\infty}$ can then be written as

$$
f_{1}^{\infty}(z)=A_{0}\left[\mathrm{e}^{-\lambda z}+\sum_{k=1}^{\infty} \frac{(-1)^{k} \alpha^{k}}{\prod_{j=1}^{k}\left(\alpha^{j}-1\right)} \mathrm{e}^{-\lambda \alpha^{k} z}\right],
$$

where $A_{0}$ is proportional to the integral of the solution $f_{1}^{\infty}$ and can be evaluated exploiting the conservation of $f_{1}^{\infty}$ ensured by Eq. (4.16)

$$
\int_{0}^{\infty} f_{1}^{\infty}(z) \mathrm{d} z=\int_{0}^{\infty} f_{1}(t=0, z) \mathrm{d} z=\bar{F} .
$$

One then has

$$
\bar{F}=A_{0}\left[\frac{1}{\lambda}+\frac{1}{\lambda} \sum_{k=1}^{\infty} \frac{(-1)^{k}}{\prod_{j=1}^{k}\left(\alpha^{j}-1\right)}\right],
$$

or

$$
A_{0}=\frac{\lambda \bar{F}}{1+\sum_{k=1}^{\infty} \frac{(-1)^{k}}{\prod_{j=1}^{k}\left(\alpha^{j}-1\right)}} .
$$

The normalized stationary state

$$
\begin{aligned}
\frac{f_{1}^{\infty}(z)}{\bar{F}}= & \frac{\lambda}{1+\sum_{k=1}^{\infty} \frac{(-1)^{k}}{\prod_{j=1}^{k}\left(\alpha^{j}-1\right)}} \mathrm{e}^{-\lambda z} \\
& +\sum_{k=1}^{\infty} \frac{(-1)^{k} \alpha^{k}}{\prod_{j=1}^{k}\left(\alpha^{j}-1\right)} \mathrm{e}^{-\lambda \alpha^{k} z},
\end{aligned}
$$

is plotted in Fig. 3 versus $Z=\lambda z$ for different values of $\alpha$. It gives the stationary distribution of potentially progressing cells, which is reached thanks to the control of the immune system and to the absence of net proliferation/death of both tumor and immune cells. Higher values of $\alpha$ correspond to an immune system more effective in controlling the progression of endothelial cells. As a consequence, the stationary solution has a peaked maximum near $z=0$. Smaller values of $\alpha$ correspond to an immune system less effective in controlling the progression of endothelial cells and the population of endothelial cells reaches a stationary configuration characterized by a higher mean progression. In fact, both $m^{\infty}$ and $\sigma^{\infty}$ decrease with $\alpha$.

Figure 4 shows how the solution computed numerically integrating Eq. (5.2) for $\alpha=10 / 7$ tends toward the analytical solution given by Eq. (5.11). The initial distribution has a compact support: in biological terms, there is initially a maximum value of progression for the cells. Due to the hyperbolic character of Eq. (5.2), this characteristic is preserved, that is at any finite time there is a maximum progression value related to the maximum initial progression and the progression velocity $c$. It can be noticed, however, that, being the asymptotic solution for $t \rightarrow+\infty$, the steady solution (5.11) has not a compact support. It is strictly positive for all $z>0$ and goes to zero exponentially for large $z$. As analytically expected, the total number of progressing cells is unchanged. They tend toward more pathological states, but are controlled by the immune system.

Case III. $\alpha=1, \nu=0, \gamma>0, \delta>0$

This case corresponds to a balance between proliferation and inhibition of immune cells and to a destruction ability of immune cells toward progressing cells. In turn, progressing cells undergo proliferation. Again $n_{2}$ is 


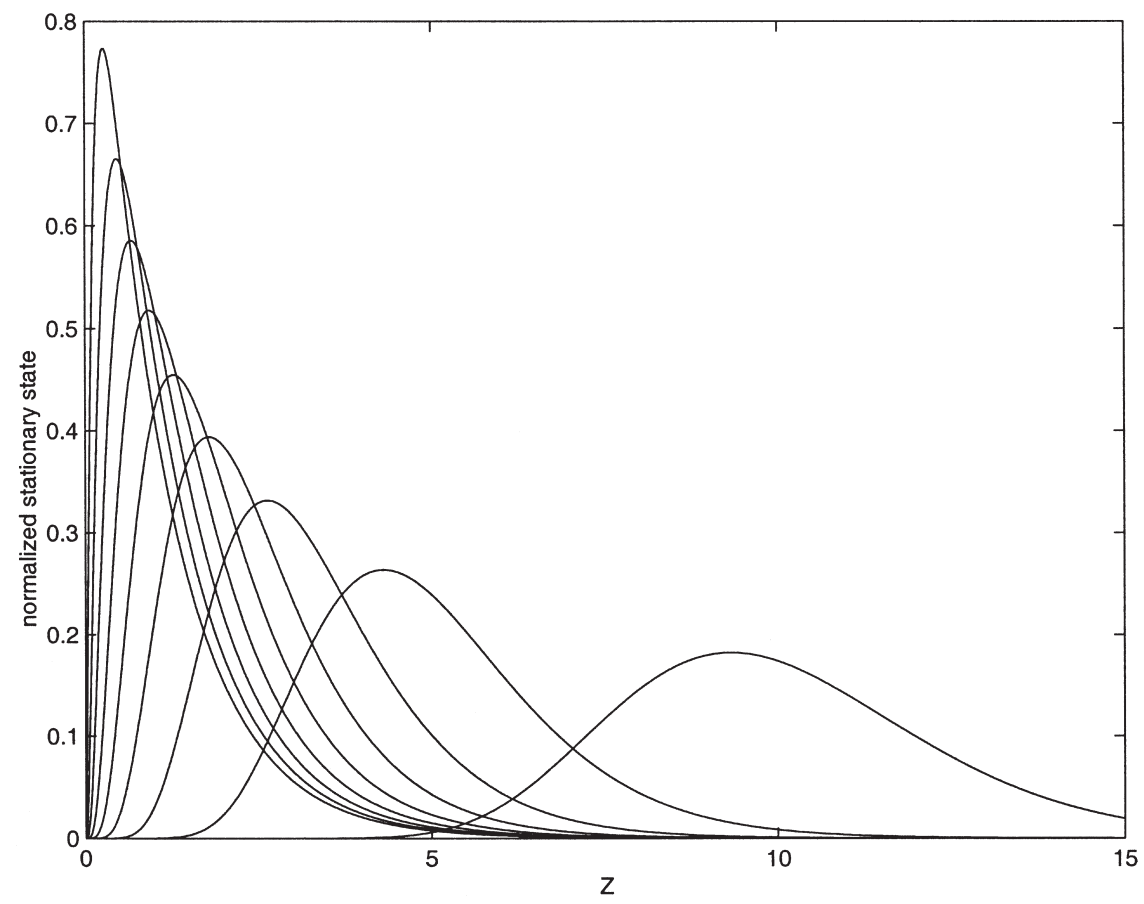

FIGURE 3 Steady states for $\alpha=10 /(10-i), i=1, \ldots, 9$. Higher values of $\alpha$ correspond to higher maxima nearer $Z=\lambda z=0$ and to immune systems more effective in controlling tumor progression.

constant, while $f_{1}$ evolves according to

$$
\frac{\partial f_{1}}{\partial t}(t, z)+c \frac{\partial f_{1}}{\partial z}(t, z)=\left(\gamma z-\delta n_{2}\right) f_{1}(t, z)
$$

which can be integrated giving for $z>c t$

$$
f_{1}(t, z)=f_{1}^{0}(z-c t) \exp \left\{\gamma z t-\delta n_{2} t-\frac{\gamma c}{2} t^{2}\right\}
$$

where $f_{1}^{0}$ is the initial condition. The solution for $z<c t$ depends on the boundary condition at $z=0$. For instance, it identically vanishes if there is no inflow of progressing cells. The discussion of the more general case with nonvanishing boundary data is similar. We will then only focus on what happens for $z \geq c t$.

Considering the characteristic line through the point $\left(t=0, z=z_{0}\right)$ with $z_{0}<\delta n_{2} / \gamma$ and such that $f_{0}\left(z_{0}\right)>0$,

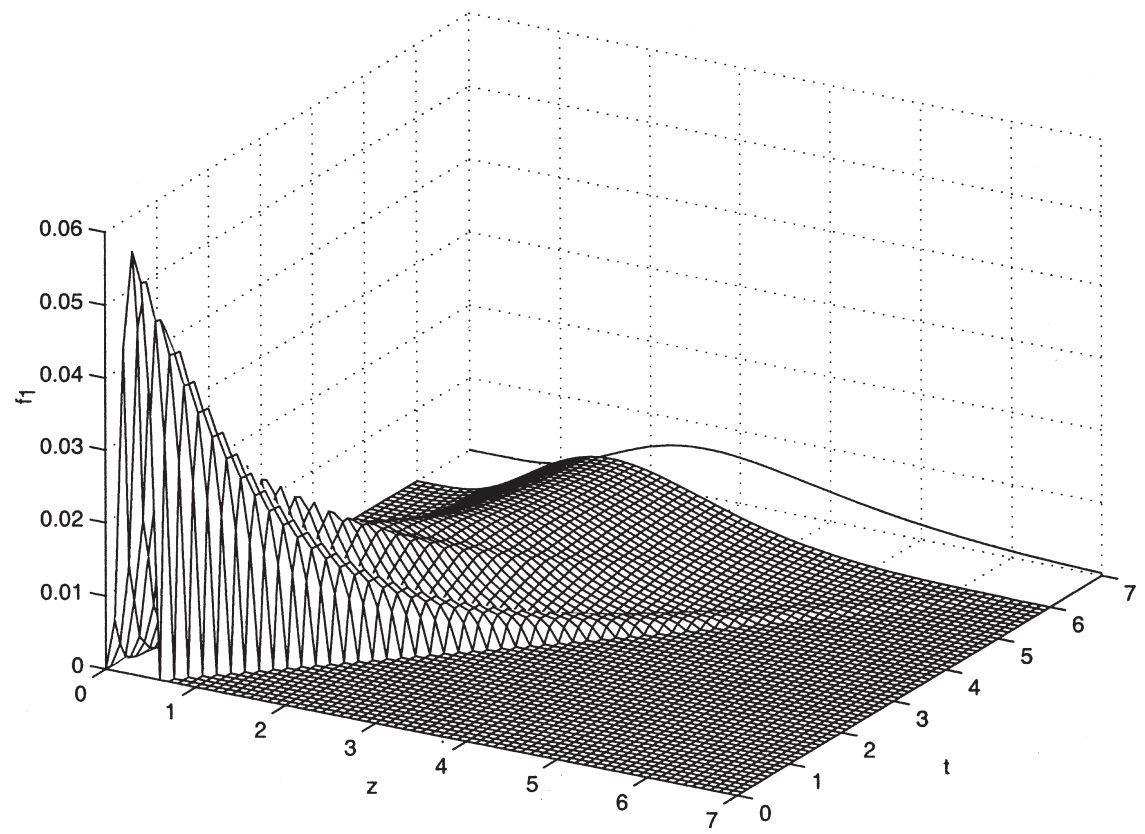

FIGURE 4 Evolution of the statistical distribution $f_{1}$ of progressing cells toward the stationary solution for $\alpha_{12}=0.3(\alpha=10 / 7)$. The line drawn for $t=7$ is the analytic solution (5.11). 


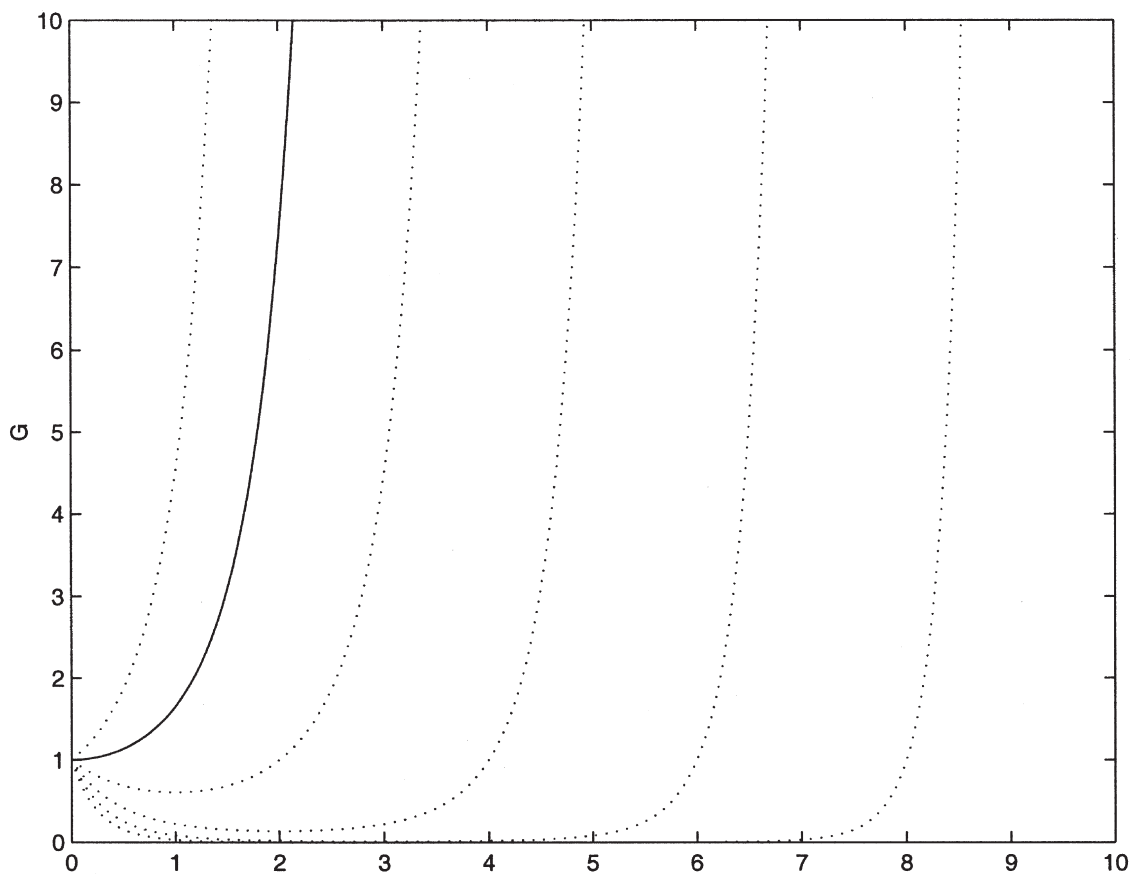

FIGURE 5 Temporal evolution of $G=f_{1}(t, z(t)) / f_{1}\left(0, z_{0}\right)$ for $\alpha=c=\gamma=1, \nu=0$ and $\delta n_{2}=4$ along the characteristics $z(t)=z_{0}+c t$. From right to left the lines correspond to $z_{0}=1,2,3,4,5$. In particular, the full line corresponds to the evolution along the characteristic through the point $z_{0}=\delta n_{2} / \gamma=4$.

the behavior of the solution along it exhibits a minimum

$$
\frac{f_{1}^{\min }}{f_{1}^{0}\left(z_{0}\right)}=\exp \left\{-\frac{\left(\delta n_{2}-\gamma z_{0}\right)^{2}}{2 \gamma c}\right\}
$$

at $t=\left(\delta n_{2}-\gamma z_{0}\right) / \gamma c$ and then an exponential growth to infinity. If from the beginning $f_{1}$ has support that goes beyond $z=\delta n_{2} / \gamma$ (i.e. if there are some cells with initial progression state above this threshold value), then from that characteristic on the solution immediately grows without passing through a minimum (see Fig. 5). This behavior characterizes also the case $\delta=0$ (even allowing $\nu \neq 0$ ). In this case the immune system has no effect on the progressing cells, though it is affected by their presence.

These features are presented in Figs. 5 and 6. In particular, Fig. 5 refers to the evolution along the characteristics and therefore on the temporal evolution of the number of cells which initially have a given progression state $z_{0}$. It can be observed that for smaller values of $z_{0}$ the tumor seems to disappear, but the remaining cells progress on becoming more and more aggressive, eventually leading to a final burst of the number of cells. Fig. 6a,b give the evolution of $f_{1}(t, z)$ in the case in which the initial support is contained or not in $\left[0, \delta n_{2} / \gamma\right]$. Note also in this case how the tumor seems to be destroyed, before the sudden growth at higher progression states.

Case IV. $\alpha>1, \gamma=0, \nu \neq 0, \delta>0$

This particular case corresponds to no proliferation of progressing cells, while the conservative and destructive action of the immune system act. In turn, the immune system is affected by the presence of progressing cells. There is no balance between proliferation and inhibition of immune cells. However, because of the absence of the growth term for the progressing cells Eqs. (4.13) and (4.16) give rise to the following closed system of ordinary differential equations

$$
\begin{gathered}
\frac{\mathrm{d} F}{\mathrm{~d} t}(t)=c b(t)-\delta n_{2}(t) F(t), \\
\frac{\mathrm{d} n_{2}}{\mathrm{~d} t}(t)=\nu n_{2}(t) F(t),
\end{gathered}
$$

which, assuming $b(t)=0$, possesses the first integral

$$
\nu F(t)+\delta n_{2}(t)=\text { const. }
$$

The equilibrium solutions present either $F$ or $n_{2}$ vanishing.

If $A=\delta n_{0}+\nu F_{0} \neq 0$, where $n_{0}$ and $F_{0}$ are the initial values of $n_{2}(t)$ and $F(t)$, the solution of Eq.(5.14) can be written as

$$
\begin{array}{r}
n_{2}(t)=\frac{A n_{0}}{\delta n_{0}+\nu F_{0} \mathrm{e}^{-A t}}, \\
F(t)=\frac{A F_{0} \mathrm{e}^{-A t}}{\delta n_{0}+\nu F_{0} \mathrm{e}^{-A t}} .
\end{array}
$$

If $\nu<-\delta n_{0} / F_{0}<0$, e.g. if the aggressive behavior of progressed cells towards the immune system or the quantity of progressing cells is strong enough, then $n_{2}(t)$ tends to zero, i.e. the immune system becomes completely 


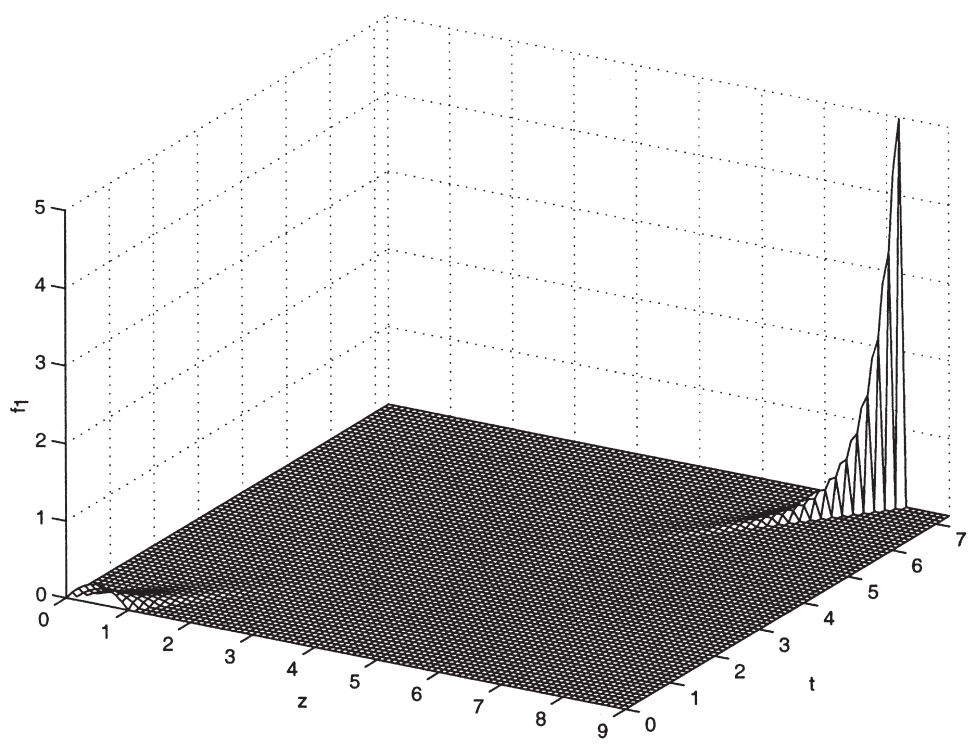

(a)

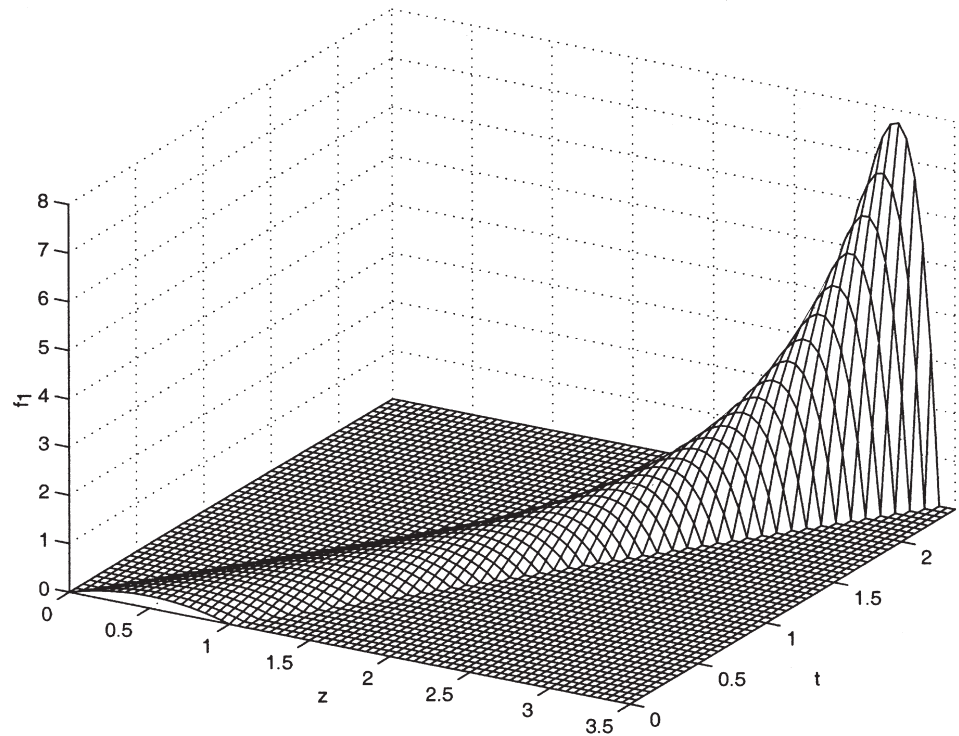

(b)

FIGURE 6 The statistical distribution $f_{1}(t, z)$ of progressing cells is plotted in (a) for the same values of the parameters as in Fig. 5 and in (b) for $\delta n_{2}=0.4$. In (b) the number of progressing cells constantly increases. In (a) it explodes after having nearly vanished.

ineffective. Otherwise, $F(t)$ tends to zero, i.e. progressed cells are destroyed. The effect of $\alpha>0$ is to slow down the progression but as it is associated to a conservative action and the progression does not influence the evolution (i.e. $\gamma=0$ ) it does not have any effect on the total number of progressing cells.

Case V. $\alpha>1, \gamma>0, \nu \neq 1, \delta=0$

In this case tumor progression is only hampered by the conservative action of the immune system. Equation (4.22) reduces to

$$
\frac{\mathrm{d} F}{\mathrm{~d} t}(t)=\gamma \int_{0}^{\infty} z f_{1}(t, z) \mathrm{d} z=\gamma m(t) F(t)>0,
$$

which states that $F$ always increases.
On the other hand, the solution of Eq. (4.13) for $\gamma>0$ is larger that the one corresponding to $\gamma=0$ dealt with in Case II. Therefore, the right hand side is always larger than a strictly positive number, which implies that $F$ goes to infinite with time and the number of progressing cells explodes.

The description of particular cases allows to enlighten, at some extent, the solution of the general case that typically could evolve according to the following scenarios.

1. The presence of progressed cells stimulates the duplication of immune cells.

2. The immune cells have a double action on progressed cells: conservative and destructive. For small progression the latter is dominant, the number of 
progressed cells decreases and the mean progression is controlled (recall the second equation in (4.13) and Case I).

3. As $F$ becomes smaller and smaller $n_{2}$ tends to a constant.

4. A few cells may reach a progression state such that the right hand side of the first equation in (4.13) returns positive. This might give raise to a renewed tumor growth and consequential immune response.

The possible dynamics pointed out by this discussion motivates the need for a more detailed study of the qualitative behavior of the solution in the general case.

A main applicative interest is in devising for which set of parameters $\alpha, \gamma, \nu, \delta, c$ the solution of Eq. (4.13) is stable in a suitable sense. As the integral of $f_{1}$, appearing in the equations is related to the number of progressed cells and some terms in the equations ensure conservation, it is quite natural to look for solutions with bounded $L^{1}$ norm, i.e. with bounded mass

$$
\left\|f_{1}\right\|_{L^{1}} \equiv \int_{0}^{\infty} f_{1}(t, z) \mathrm{d} z \equiv F(t)<M
$$

where $M$ is independent of time.

A conceivable additional investigation item is to check whether for some values of the parameters $F$ tends to zero. However, from a biological point of view the knowledge of such a behavior provides only a partial answer. The critical time at which the tumor mass starts to decrease depends on the parameters of the model. Of course, the critical time could be much longer than the human life. Moreover, the function $F$ should tend to zero without passing through maximum values not compatible with the survival of the organism. According to the parameters, such a value can be overcome even if the model foresees a theoretical asymptotic damping of $F$ for very large times.

\section{CRITICAL ANALYSIS AND RESEARCH PERSPECTIVES}

The contents of this paper were developed with direct reference to the paper by Greller et al. (1996), which shows that in describing the evolution of a cell population towards pathological states it is necessary to introduce a new independent variable which describes the different behavior of cells according to their progression states. Indeed, a general framework and related models have been proposed to describe in mathematical terms most of the phenomena phenomenologically described in Greller et al. (1996) and characterized by the fact that the behavior at a certain time and position depends not only on the number of cells involved, but also on their progression states.

In some particular regimes, the model shows a simple enough structure to allow the determination of analytic solutions and asymptotic behavior. The analysis of the above simplified models provides useful information on the asymptotic behavior in the general case.

The general framework proposed in "A conceptual modeling framework" section can be further developed in order to describe some interesting phenomena which have not been explicitly considered in this paper. For instance, the reproduction rate is here assumed to be proportional to the progression state. On the other hand, in situations like chronic myelogenous leukemia, the growth rate is nearly constant for progression values less than a certain value (giving rise to a long phase in which the tumor is controlled and physiologically tolerated) and then rapidly increases when this threshold value is reached (giving rise to an acute and probably mortal phase).

Moreover, non constant growth terms would also give rise to phenomena like clonal dominance. In fact, those states characterized by a larger growth coefficient would eventually be dominant. Clonal dominance can also be described by a progression velocity that vanishes at a given value of progression. This would cause a crowding of the characteristics towards this asymptotic value.

The production of immune cells by the bone marrow can be modeled as an external source term in the equation for the immune system. This term can be constant, providing a constant input or might be time dependent, modeling the possibility to stimulate pharmacologically the production of immune cells. Further, medical actions can be modeled operating on the left-hand-side term as in Firmani et al. (1999).

Looking again at Greller et al. (1996), it results that in some cases the introduction of a single progression state is not sufficient, but more progression states are to be defined. In fact, the framework developed in this paper allows to deal only with those cases in which different ordering of two distinguishable genetic events lead to the same tumor state. Introducing at least two progression variables $u_{1}$ and $u_{2}$ would allow to explain situations in which the ordering of the genetic events leads to different dynamics because of different paths in the progression plane $\left(u_{1}, u_{2}\right)$.

It is plain that several additional phenomena can be taken into account. Listing them would not be difficult and some of them are also outlined in Greller et al. (1996). The difficulty is mainly in providing a consistent mathematical description. In some cases, the development of the model of this paper may be simply a technical problem, in other cases the development can be obtained only by a deep analysis of the system.

In principle, the modeling may even require the modification of the general structure of the equations proposed in "A conceptual modeling framework" section. Indeed, we think that this is going to be an interesting field of research and speculations for applied mathematicians. A further relevant aspect refers to the analysis of the connections between the microscopic description, developed at a cellular level, proposed in this paper and the macroscopic description developed starting by suitable 
conservation equations (see for instance De Angelis and Preziosi (2000)). In fact, the framework presented here does not take space and cell motion into account and this has to be done to relate these models with classical reaction-diffusion models. On the other hand, it is clear from the phenomenological description given by Greller et al. (1996) that the macroscopic evolution of a tumor depends on the progression state of its cells and therefore reaction-diffusion models should take into account that progression is a statistically distributed characteristic of cells influencing their global behavior. In this respect, this paper should be considered as a first step toward the above research program.

\section{Acknowledgements}

This research has been partially supported by the European Community through a Research Training Network Programme on Using Mathematical Modeling and Computer Simulation to Improve Cancer Therapy, by the Consiglio Nazionale delle Ricerche under a contract on Mathematical Modeling of Biological Systems, and the Ministry of University, Scientific and Technological Research through a project on Modeling and Mathematical Methods to Support Cancer Research.

\section{References}

Forni, G., Foa, R., Santoni, A., Frati, A., (1996) In: A Survey of Models on Tumor Immune Systems Dynamics (Birkhäuser, Boston).

Arlotti, L., Bellomo, N. and Lachowicz, M. (2000) "Kinetic equations modelling population dynamics", Transp. Theory Statist. Phys., 29.
Arlotti, L., Bellomo, N. and Latrach, K. (1999) "From the Jager and Segel model to kinetic population dynamics nonlinear evolution problems and applications", Math. Comput. Modelling 30, 15-40.

Forni, G., Foa, R., Santoni, A., Frati, A., (1995) In: Dynamical DiseaseMathematical Analysis of Human Illness (American Institute of Physics Press, Williston).

Bellomo, N. and De Angelis, E. (1998) "Strategies of applied mathematics towards an immuno mathematical theory on tumors and immune system interactions", Math. Models Meth. Appl. Sci. 8, $1403-1429$.

Bellomo, N. and Forni, G. (1994) "Dynamics of tumor interaction with the host immune system", Math. Comput. Modelling 20, 107-122.

Bellomo, N. and Lo Schiavo, M. (1998) "From the Boltzmann equation to generalized kinetic models in applied sciences", Math. Comput. Modelling 27, 43-47.

Bellomo, N., Preziosi, L. and Forni, G. (1996) "On a kinetic (cellular) theory of the competition between tumors and the immune host system", J. Biol. Syst. 4, 479-502.

Chaplain, M. (1999) "Special issue on mathematical models for the growth, development and treatment of tumors", Math. Models Meth. Appl. Sci. 9, 491-626.

De Angelis, E. and Preziosi, L. (2000) "Advection-diffusion models for solid tumour evolution in vivo and related free boundary problems", Math. Models Meth. Appl. Sci. 10, 379-407.

Firmani, B., Guerri, L. and Preziosi, L. (1999) "Tumor/immune system competition with medically induced activation/disactivation", Math. Models Meth. Appl. Sci. 9, 491-512.

Forni, G., Foa, R., Santoni, A., Frati, A., (1994) In: Cytokine Induced Tumor Immunogeneticity (Academic Press, New York).

Greller, L., Tobin, F. and Poste, G. (1996) "Tumor hetereogenity and progression: conceptual foundation for modeling", Invasion and Metastasis 16, 177-208.

Gyllenberg, M. and Webbs, G. (1990) "A nonlinear structured population model of tumor growth with quiescence", J. Math. Biol. 28, 671-694.

Lo Schiavo, M. (1996) "Discrete kinetic cellular models for tumor immune system interactions", Math. Models Meth. Appl. Sci. 8, $1187-1210$.

Owen, M. and Sherrat, J. (1999) "Mathematical modelling of macrophages dynamics in tumours", Math. Models Meth. Appl. Sci. 9, 513-540.

Perelson, A.S. and Weisbuch, G. (1997) "Immunology for physicists", Rev. Mod. Phys. 69, 1219-1267. 


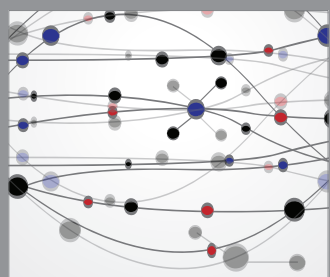

The Scientific World Journal
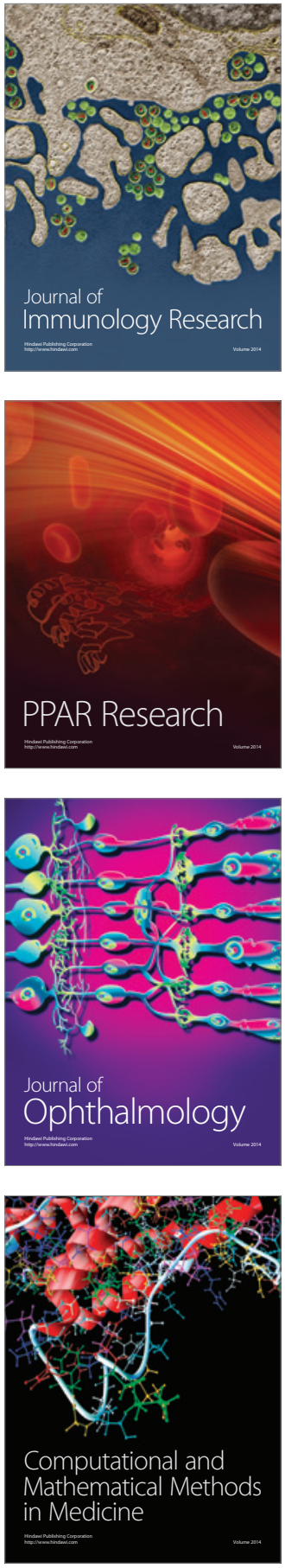

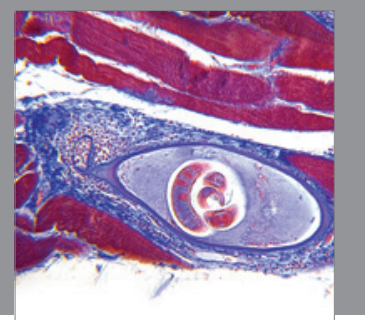

Gastroenterology

Research and Practice
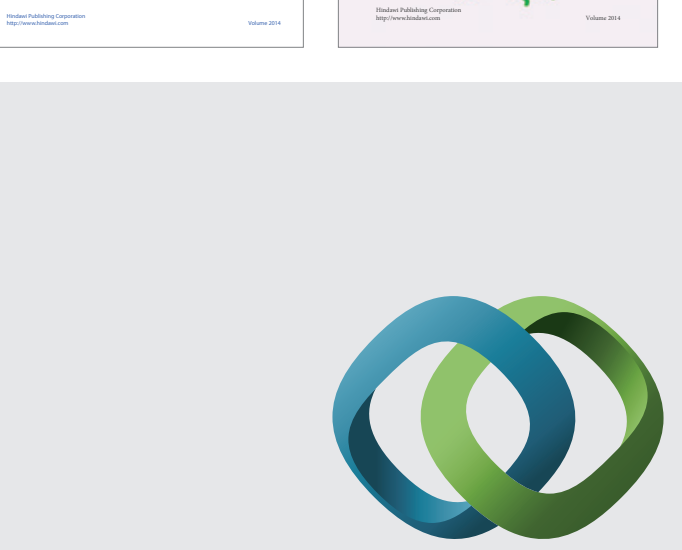

\section{Hindawi}

Submit your manuscripts at

http://www.hindawi.com
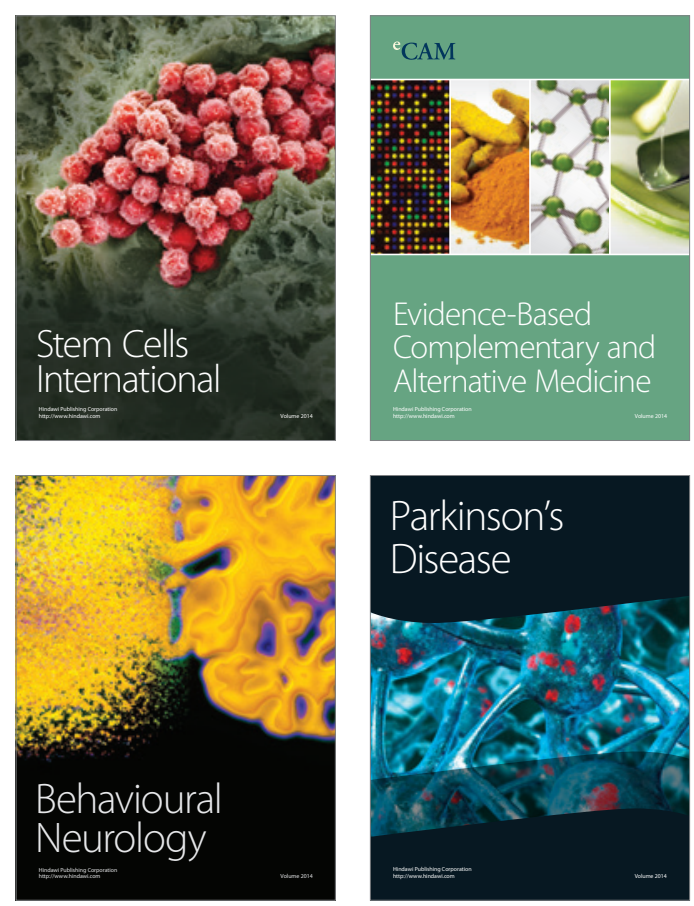

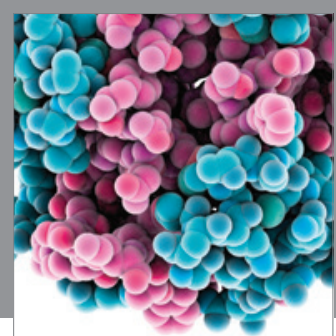

Journal of
Diabetes Research

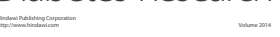

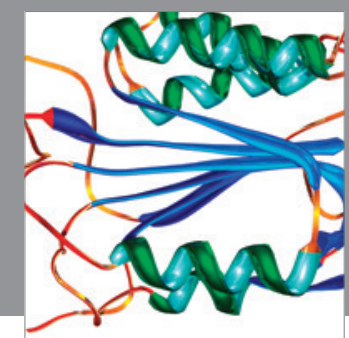

Disease Markers
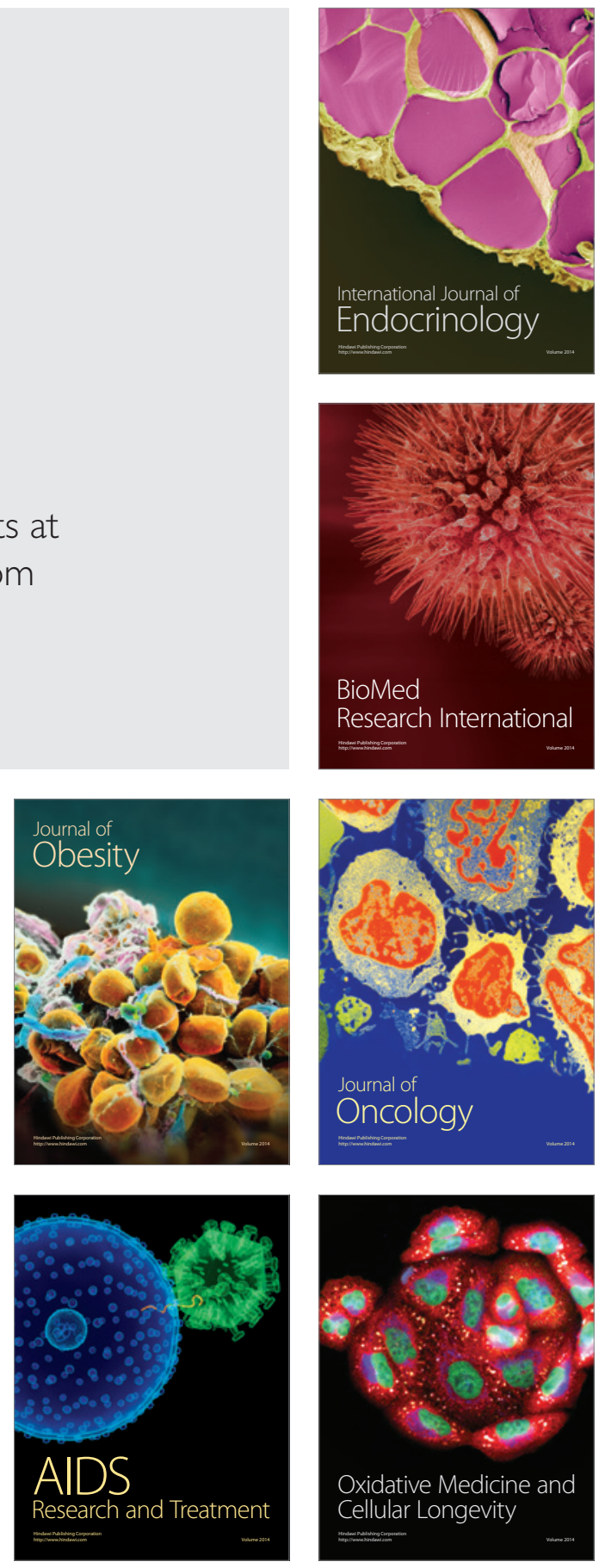